\title{
Mechanisms and applications of vibration energy harvesting in solid rocket motors
}

\author{
Chirag Goel $^{1}$ (D) $\cdot$ G. Srinivas $^{2}$ (D)
}

Received: 26 October 2020 / Accepted: 17 December 2020/Published online: 8 February 2021

(C) The Author(s) 2021

\begin{abstract}
Energy harvesting has become a fascinating topic of research. As the world moves towards reducing its dependency on fossil fuels, new and innovative techniques of energy harvesting have been tested and developed. The use of piezoelectric materials to harvest the ambient vibrations from the surroundings is one method that has seen a dramatic rise in use for power harvesting. Remote sensors can be powered by these piezoelectric materials and could potentially act as a continuous source of energy. In space vehicles, energy is generated using solar panels which are bulky, heavy and expensive. Instead piezoelectric harvesters can be used to generate power and are much lighter, compact and relatively cheap when produced in bulk. This paper presents a theoretical study on energy harvesting from structural vibration caused by combustion instability of a solid rocket motor through the motor burnout. Vibration data of tested solid rocket motor was taken as a reference and was inputted as the boundary condition. The 3-D model of the harvester system was designed on Fusion360 and the simulation was performed on COMSOL. Finally, improvements needed in the system to enhance practicality were discussed.
\end{abstract}

\section{Introduction}

In years energy harvesting has seen a rise in research and implementation. Vibration energy harvesting with piezoelectric materials is of practical interest because of the demand for wireless sensing devices and low-power portable electronics without external power supply. Piezoelectric effect is the ability of certain materials to generate an electric charge in response to applied mechanical stress. Many non-traditional energy harvesters like thermometric generator, piezoelectric transducers, solar photo voltaic cells, electromagnetic inductor coils, have come up and are being developed for mass usage (Beeby and White 2010). These energy harvesters have several advantages over traditional batteries; energy harvesters can be used to power sensors and other low voltage

G. Srinivas

srinivas.g@manipal.edu

1 Department of Mechanical and Manufacturing Engineering, Manipal Institute of Technology, Manipal Academy of Higher Education, Manipal 576104, India

2 Department of Aeronautical and Automobile Engineering, Manipal Institute of Technology, Manipal Academy of Higher Education, Manipal 576104, India electronic systems like embedded systems and IoT (Internet of Things) in remote places where the use of batteries is costly, unfeasible, or unsafe. Energy harvesters can also act as a permanent source of energy to power electronic devices and removes the electronic devices dependency on batteries (Safaei et al. 2019). Energy harvesters can supply nearly unlimited operating life to the device, making the device self-sustaining. This concept can be applied to harvest energy in rockets and space vehicles. Even though there has been a lot of research done in the improving the energy harvesting technology, not much have been done in implementation of this technology in aerospace industry.

The phenomenon of combustion instability in SRM is an oscillatory interaction between gas flow and combustion of the propellant in such a way that pressure oscillations develop with amplitudes comparable to the mean pressure (Price 1959). This instability produces longitudinal structural vibrations which travels through the whole rocket fuselage. These vibrations are generally considered as destructive but can be used to generate energy. These piezoelectric harvesters can also dampen down the vibration and can prevent structural failure of the rocket (Makihara et al. 2006). Makhihara and Shimose (Makihara and Shimose 2012) presented harvesting of electrical energy using fluttering phenomena of wings of a sounding 
rocket. In this paper, the concept of using the structural vibration in an SRM for harvesting of electrical energy is studied. A Piezoelectric energy harvester was designed for the range of frequency of the vibration at a point on the SRM. This harvested energy can then be rectified and stored to run remote sensors around the SRM.

\section{Combustion instability in a solid rocket motor}

Solid rocket motors (or SRM) are a types of rocket engine that uses solid propellant for combustion. SRMs have been used as booster stage for the space shuttle, in missiles and sounding rockets. They use various types of solid propellant like double-base (DB) propellants, composite propellants, 'Candy' propellants, etc are used by sounding rockets. Propellant are then moulded into segments and are then loaded into the combustion chamber. After the ignition of the motor, the flow of gases remains smooth before it starts to oscillates. This flow oscillation is due to combustion instability that occurs inside the combustion chamber and can cause motor failure. There are two types of combustion instability that occurs in a solid rocket motor: Pressure oscillations and Vortex shedding. Pressure oscillations occurs in all kind of motors and Vortex shedding occurs in some particular type of grains as given in Sutton and Biblarz (2017).

\subsection{Pressure oscillation}

During an unstable combustion the pressure oscillates in the range of $5 \%$ and up to $30 \%$ of the maximum chamber pressure (Sutton and Biblarz 2017). The origin of pressure oscillations may be some perturbation in the combustion chamber which gets amplified due to instantaneous increase in burn rate, chamber pressure and the thrust. The increased combustion properties can cause change in trajectory of the rocket and may lead to the failure of the mission. Combustion instability can occur spontaneously, often at some particular time during the motor burn period, and it repeats in identical motors. The frequency of the instability is function of the cavity geometry, propellant composition, pressure, and internal flame field. The pressure-coupled response of the instability is mathematically defined as the ratio between the perturbed burning rate over the mean burning rate to the perturbed pressure over the mean chamber pressure (Blomshield 2007):

$R_{p}=\frac{\mathfrak{r} / \overline{\boldsymbol{r}}}{\mathbf{p} / \overline{\boldsymbol{p}}}$.

$R_{p}$ is a non-dimensional quantity and gives the combustion response of the propellant. $R_{p}$ is a function of burning rate, pressure, frequency and propellant physical and mechanical properties.

\subsection{Vortex-shedding instability}

Vortex shedding instability is associated with the burning of the inner surface of the grain segments. Large solid rocket motor generally have multiple grain segments which have exposed inner surfaces, the gap in between two segments is known as a slot. The gases produced by burning of the inner surfaces of the segments mixes with the main flow from the central cavity of the grain geometry. This phenomena causes the upstream port pressure to rise; sometimes there is a substantial pressure rise. The interaction of the two subsonic gas flows causes turbulence. Vortices form and are periodically shed or allowed to flow downstream, thereby causing an unstable flow pattern as shown in Fig. 1 (Sutton and Biblarz 2017).

\subsection{Measurement of combustion instability}

The standard way to measure the combustion response is by using a T-burner (Blomshield 2007). T-burner is used as a standardised test to compare the responses of different propellants. Various combustion response parameters like frequency and amplitude changes with the SRM structural characteristics, Therefore the measurement of combustion response of different motors can only be done by static fire test. Taherinezhad and Zarepour (2020) did an in-depth investigation of the combustion instability phenomena using a small-scale solid rocket motor with outside diameter $122 \mathrm{~mm}$, length $1270 \mathrm{~mm}$ and throat diameter $45 \mathrm{~mm}$. Their experiment demonstrated that frequency of instability oscillations nearly equals to axial acoustic mode of the motor. The dominant frequency obtained at different distances from the head end by them is given by Table 1 (Taherinezhad and Zarepour 2020)

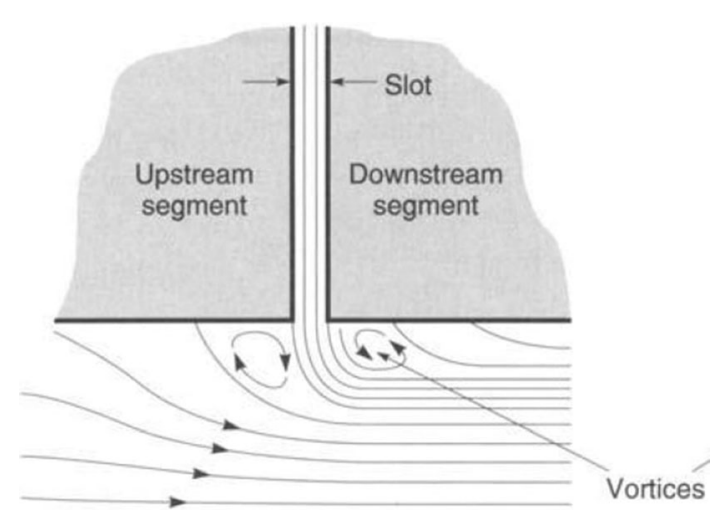

Fig. 1 Vortex shedding due to slot between two grain segments (Sutton and Biblarz 2017) 
Table 1 Dominant Frequency [12]

\begin{tabular}{lllll}
\hline Position & Head end distance $(\mathrm{mm})$ & \multicolumn{4}{l}{ Dominant frequency $(\mathrm{Hz})$} \\
\cline { 3 - 5 } & & Numerical solution & Analytical solution & Test \\
\hline 1 & 570 & 1041 & 748 & $800-900$ \\
2 & 855 & 510 & 360 & $300-400$ \\
\hline
\end{tabular}

\section{Mathematical model}

For the Mathematical Model of the piezoelectric harvesters, It is assumed to be a cantiliver beam with one end fixed and the other end free. The solution for the eigenfrequency of the beam is calculated using Euler-Bernoulli Beam equation which was given by Gere and Timoshenko (1997). The beam is Assumed to be uniform and has its cross-section always remains plane. The dynamic equation of motion by Euler Bernoulli equation for a homogeneous beam is given by Eq. (2) (Gere and Timoshenko 1997).

$E I \frac{\partial^{4} w}{\partial x^{4}}=-\mu \frac{\partial^{2} w}{\partial t^{2}}+q(x)$

This equation in the case of free vibration have no external loading $\mathrm{q}(\mathrm{x})$. This Equation can be solved by using Fourier decomposition of displacement into sum of harmonic vibration.

$w(x, t)=\operatorname{Re}\left[\hat{w}(x) e^{-i \omega t}\right]$.

The solution of the above equation is

$\hat{w}_{n}=A_{1} \cosh \left(\beta_{n} x\right)+A_{2} \sinh \left(\beta_{n} x\right)+A_{3} \cos \left(\beta_{n} x\right)+A_{4} \sin \left(\beta_{n} x\right)$

where $\beta_{n}:=\left(\frac{\mu \omega_{n}^{2}}{E I}\right)^{1 / 4}$

The above Eq. (5) represents the natural frequency of the beam. $\beta$ can be calculated subjected to different boundary conditions. For this research, boundary condition for a cantiliver beam is used:

$$
\begin{array}{cc}
\hat{w}_{n}=0, \frac{d \hat{w}_{n}}{d x}=0 \quad \text { at } x=0 \\
\frac{d^{2} \hat{w}_{n}}{d x^{2}}=0, \frac{d^{3} \hat{w}_{n}}{d x^{3}}=0 \quad \text { at } x=L .
\end{array}
$$

Solution for the Eq. (4) is non-trivial only if

$\cosh \left(\beta_{n} L\right) \cos \left(\beta_{n} L\right)+1=0$.

This equation is solved using numerical methods and various modes of natural frequency can be calculated.

The First Natural frequency is:
$\frac{1.875^{2}}{L^{2}} \sqrt{\frac{E I}{\mu}}$.

The Second Natural frequency is:

$\frac{4.694^{2}}{L^{2}} \sqrt{\frac{E I}{\mu}}$.

From the above equations it is clear that the natural frequency of the beam depends directly on the square root of flexural rigidity (EI) and inversely proportional to the square of the length of the beam.

If piezoelectric materials are physically deformed by pressure or vibration, it creates an induced electrical field across it. In contrast, if electrical energy is applied, then mechanical deformation will induce. The piezoelectric cantilever electrical charge density can be calculated using Eq. (10).

$S_{1}=s_{11}^{E} T_{1}+d_{31} E_{3}$
$D_{3}=d_{31} T_{1}+\varepsilon_{33}^{T} E_{3}$

Where, piezoelectric material's stress (T), strain (S), charge-density displacement (D), and electric field (E) interact. The matrix $\mathrm{d}$ contains the piezoelectric coefficients for the material.

\section{Energy conversion}

The vibration on the fixed end of the beam will produce an oscillating mechanical load on the beam. This mechanical load produces stress on the cantiliver beam which gets converted to electrical energy because of the piezoelectric effect. Therefore, The output current and voltage of the piezoelectric energy harvester will be alternating in nature. However, most devices use DC power supply and the short-time output energy of the harvester will too low to directly drive microelectronic devices. Therefore, the effective method for supply energy is to use rectifier circuit and storage devices as an intermediate link, which converts alternating current into direct current, accumulates and stores the energy, then supplies power to the microelectronic devices. Energy storage and electronic circuits for energy harvesting are well studied and defined (Priya and Inman 2010). 


\section{Harvesting system}

The most commonly used shape in piezoelectric energy harvesting is the cantilever beam, as this structure creates the highest average strain for a given input force (Xu 2016). Cantilever-type energy harvesters consists of a thin cantilever beam with a proof mass at its free end and a fixed base at the other end. The entire structure is mechanically attached to a vibrating body via the fixed base. Piezoelectric cantilever beam harvesters can be divided into two types. One is the unimorph-type, which is one piece of thin piezoelectric plate bonded on one side of an elastic beam. The other is bimorph-type, which is two pieces of piezoelectric plates bonded on both sides of an elastic beam. In this design, A bimorph-type piezoelectric harvester is used. The material used are copper and PZT$5 \mathrm{H}$. Copper is the substrate and PZT-5H is the piezoelectric material. The properties of the material are shown in the Table 2 (Uddin et al. 2016; Kulkarni et al. 2017).

\subsection{Design of the harvester}

Maximum energy is harvested by the piezoelectric harvester at its resonant frequency, therefore the harvester should be tuned to the frequency of vibration. The frequency of oscillation varies with the distance from the motor head end as shown by Taherinezhad and Zarepour (2020). Therefore to take the maximum advantage of the oscillating SRM, multiple harvesters needs to be attached and tuned to the frequency of the SRM at the point of attachment. Since the frequency of oscillations at any position of a SRM is not constant, a beam whose resonant frequency lie within the dominant frequency range at that position was designed. The resonant frequency of the developed beam is $450 \mathrm{~Hz}$ and can be fixed at position 2 (Table 2). The Dimensions of the harvester are given in the Table 3.

For modelling of the harvester, Assumptions given by Kumar et al. (2016) is used. Copper is used as the substrate material as it generates the highest power for a fixed-geometry cantilever beam. Two spoof masses of mass were placed at the free end of the harvester each of mass $1.22 \mathrm{~g}$.

Table 2 Material properties

\begin{tabular}{lll}
\hline Properties & Materials \\
\cline { 2 - 3 } & PZT-5H & Copper \\
\hline Youngs modulus $(\mathrm{Pa})$ & $62 \times 10^{9}$ & $124 \times 10^{9}$ \\
Poissons ratio & 0.31 & 0.33 \\
Density $\left(\mathrm{kg} / \mathrm{m}^{3}\right)$ & 7500 & 2700 \\
\hline
\end{tabular}

Table 3 Dimensions of the harvester

\begin{tabular}{llll}
\hline & Thickness $(\mathrm{mm})$ & Width $(\mathrm{mm})$ & Length $(\mathrm{mm})$ \\
\hline PZT layer & 0.19 & 12.70 & 31.75 \\
Substrate layer & 1.5 & 12.7 & 50 \\
\hline
\end{tabular}

The CAD was designed on Fusion360 software and is represented by Fig. 2.

\subsection{Simulation}

The simulation the system was performed on COMSOL Multiphysics software. 3-D CAD file was imported to the software to analyse the mechanical and electrical coupling properties. For solid mechanics, the materials were assigned to each component of the CAD. The top and bottom piezoelectric layer were assigned PZT material, the substrate was assigned copper material and steel was assigned to the two spoof masses. Fixed constraints was applied to base part with two perforation. Body load was also applied on the whole geometry. For the sake of simplicity no damping was assumed.

For the Electrostatics,layers in contact with the substrate was used as ground and the other two surfaces were used as terminals. The electrostatics physics and electrical circuit physics were coupled to obtain the electrical response. Tetrahedral mesh with normal element size was used to discritize of the beam as shown by Fig. 3. Eigen frequency analysis was performed to determine the natural frequency of the beam. Frequency domain analyses was conducted on the geometry to get the output dependency against acceleration and frequency. For frequency domain, frequency range from 400 to $500 \mathrm{~Hz}$ with a step of $5 \mathrm{~Hz}$ was studied and since the acceleration of the oscillation vary in a

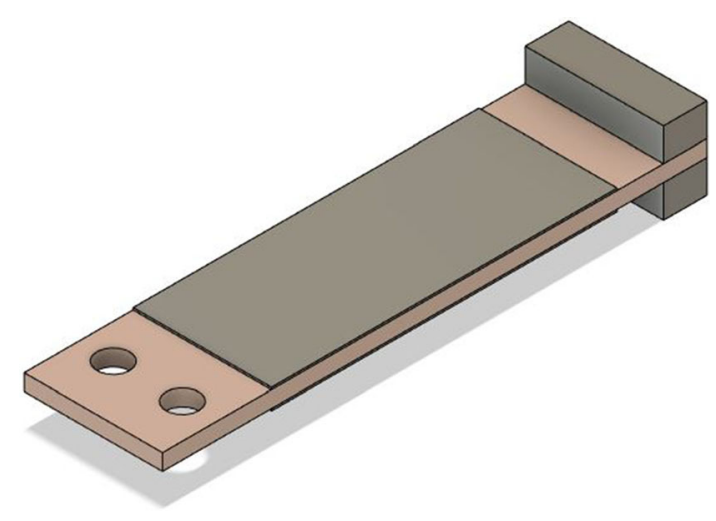

Fig. 2 3-D model of the harvester 


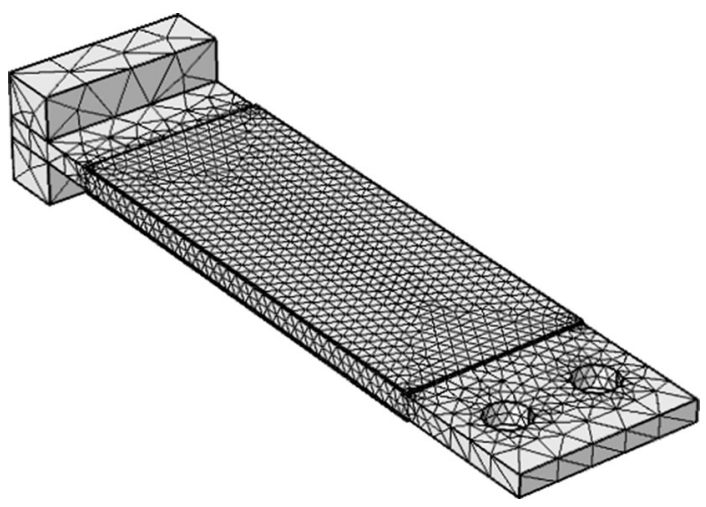

Fig. 3 Finite element mesh of the beam

practical conditions, acceleration range from 1 to $10 \mathrm{~g}$ was studied.

\section{Results}

The energy conversion from the mechanical energy to electrical energy will maximum when the piezoelectric cantilever beam will be driven at its resonant frequency. Mechanical properties of the beam such as displacement and stress were analysed with respect to frequency. The first natural frequency obtained for the presented harvester was $450 \mathrm{~Hz}$.

Figure 4 shows the stress developed across the beam at the resonant frequency of $450 \mathrm{~Hz}$. The maximum stress developed was 7.37 Mpa near the fixed clamped base. The stress in the beam decreases with the length. The electric charge generated increased with the stress, therefore electric charge decreases with the increase in length from the base.

Figure 5 shows the $\mathrm{Z}$ axis displacement of the beam. Maximum displacement of $20 \mu \mathrm{m}$ was observed at the free

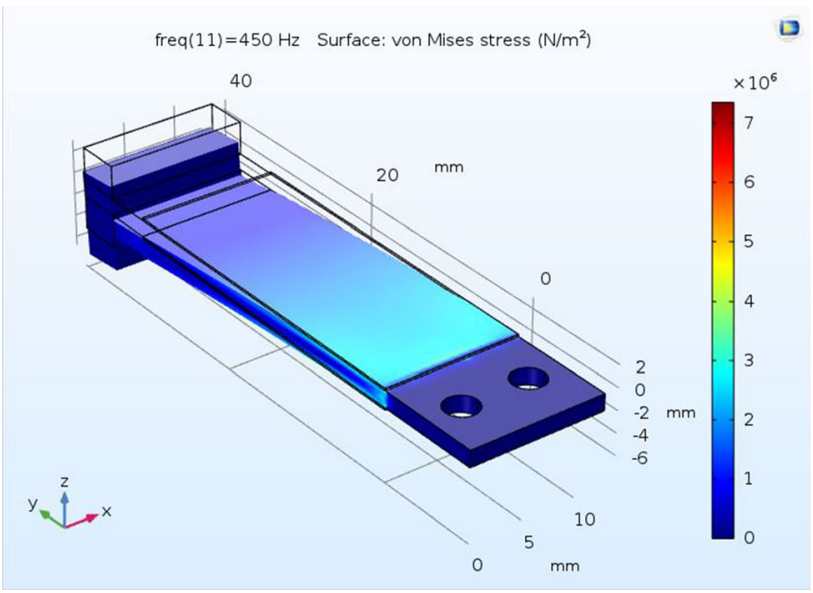

Fig. 4 Stress developed in the beam at resonant frequency

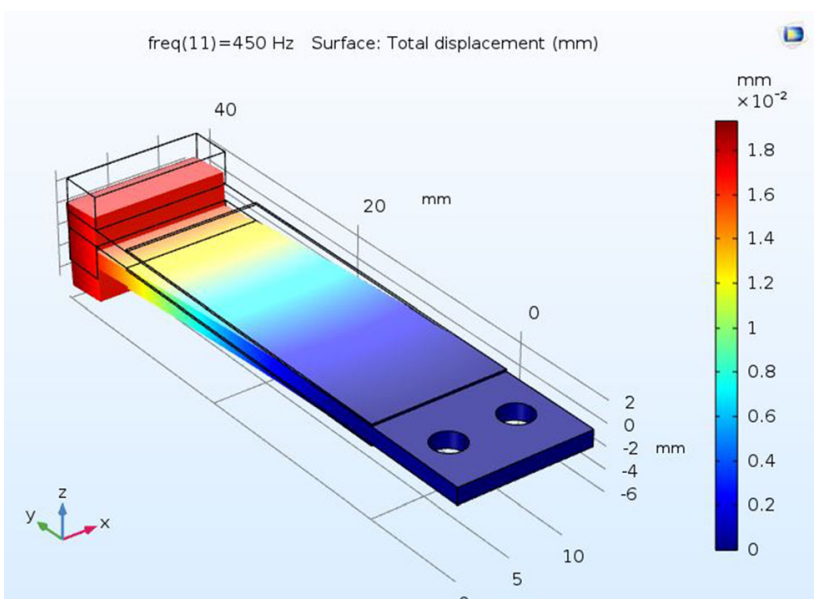

Fig. 5 Vertical displacement of the beam at resonant frequency

end. This displacement decreases with the shift in the driving vibration frequency from the resonance.

Figure 6 shows the bell curve of the electrical output and it can be seen that the output is maximum at resonance. The harvester produced a max voltage output of $11.72 \mathrm{~V}$, power output of $5.725 \mathrm{~mW}$ and current output of $0.978 \mathrm{~mA}$ across a resistance of $12 \mathrm{k} \Omega$ at $1 \mathrm{~g}$ acceleration. The output will decrease as the oscillating frequency deviates from the resonant frequency.

Figure 7 shows the dependence of the electrical output on the acceleration of vibration. Acceleration dependency from 1 to $10 \mathrm{~g}$ was studied. The voltage increased from 11.72 to $127.9 \mathrm{~V}$, power from 5.725 to $681.5 \mathrm{~mW}$ and current from 0.978 to $10.65 \mathrm{~mA}$.

$\square$

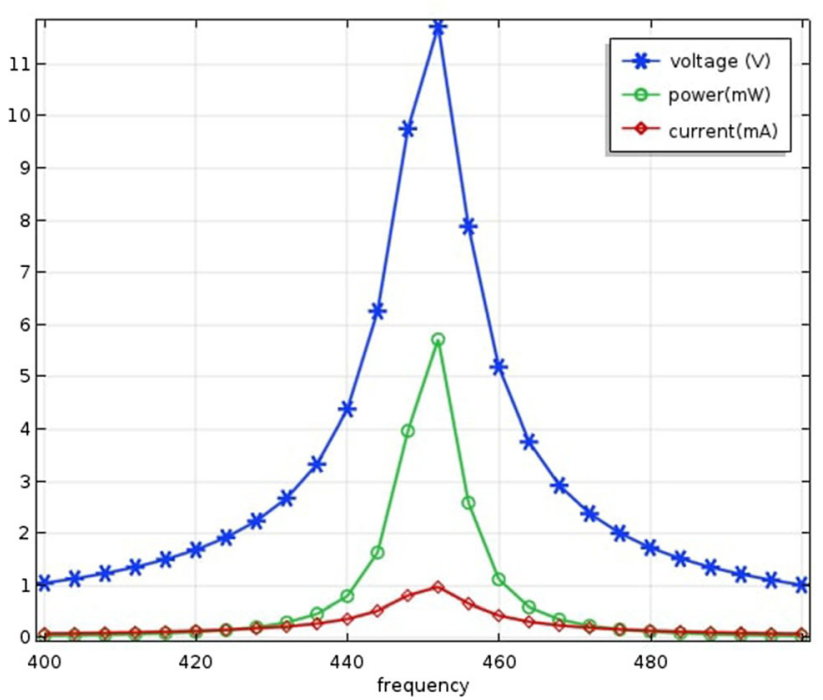

Fig. 6 Voltage, current, power vs frequency 


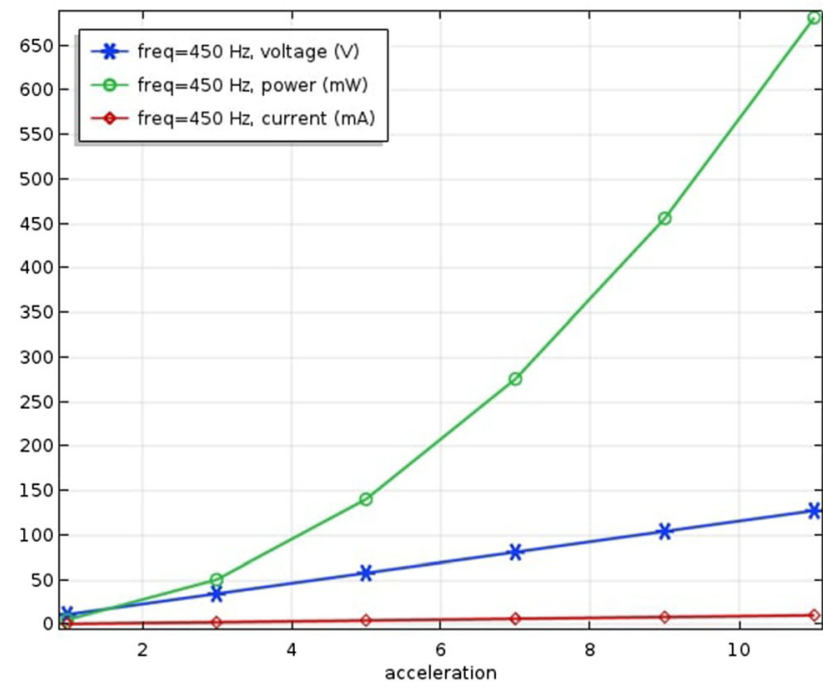

Fig. 7 Voltage, current, power vs acceleration

\section{Discussion}

The described system can be used to harvest energy at only one position in an SRM. Since, the SRMs are symmetrical these harvesters can be fixed around the circumference. This will proportionally increase the net power generated. This system can be improved by using a self tuning piezoelectric harvester. This will save time and money required for manufacturing and development of harvester with a fixed resonance frequency, this method will also improve the net energy output. An array of self tuning piezoelectric harvesters can be placed along the whole length of the motor, from the motor head end to the nozzle. This could maximize the energy generation and reduce the oscillating structural load on the fuselage of the rocket.

Further research is required in the field of additive manufacturing and material sciences to rapidly produce piezoelectric materials with excellent performance, high energy storage density, and high electromechanical conversion efficiency.

In this paper only phenomena of oscillations due to combustion instability in engines using solid propellant was discussed. This study can be expanded to liquid and hybrid type propulsion system. Phenomena such as Helmholtz, acoustic and pressure node are dominant causes of the combustion instability in liquid and hybrid. Mathematical modelling and simulation will be required to determine the properties of the structural oscillations produced and for the selection of position for harvesters placement.

\section{Conclusion}

An energy harvesting system that extracts energy from the combustion instability of a solid rocket motor was presented in this paper. This study employed the phenomena of vortex shedding and pressure oscillations which produces combustion instability in solid rocket motors. COMSOL Multiphysics software was used for the simulation the piezoelectric harvester. Our proposed harvesting system generated $5.725 \mathrm{~mW}$ of energy at $450 \mathrm{~Hz}$ across a resistance of $12 \mathrm{k} \Omega$ at $1 \mathrm{~g}$ acceleration. This technology needs to be further refined and tested in rockets. It is expected that in future the proposed application will be taken as a consideration while designing rocket motors. It is anticipated that this study will be the basis for further experimentation. More experimental validation is essential for assessing the harvesting performances. The effective utilization of combustion instability changes our perception of it from being a destructive phenomenon to being useful in various applications.

Acknowledgements This research was supported by ThrustMIT. We thank our colleagues at ThrustMIT for providing their insight and expertise that greatly assisted this research. We would like to thank Manipal Academy of Higher Education, Manipal for their assistance.

Author Contributions Chirag Goel carried out designing, data collection, simulation and writing of the manuscript. Srinivas G participated in research coordination. The authors read and approved the final manuscript.

Funding Open access funding provided by Manipal Academy of Higher Education, Manipal. No Funding was required to carry out this research.

Data Availibility Statement All data sets used to conduct this research is open access and can be accessed by DOI 10.1016/j.cja.2019.11.010.

\section{Compliance with ethical standards}

Conflict of interest The authors declare that they have no conflict of interest.

Code availability This research used Fusion360 and COMSOL software. Access to both the software was provided by Manipal Institute of Technology, Manipal.

Open Access This article is licensed under a Creative Commons Attribution 4.0 International License, which permits use, sharing, adaptation, distribution and reproduction in any medium or format, as long as you give appropriate credit to the original author(s) and the source, provide a link to the Creative Commons licence, and indicate if changes were made. The images or other third party material in this article are included in the article's Creative Commons licence, unless indicated otherwise in a credit line to the material. If material is not included in the article's Creative Commons licence and your intended use is not permitted by statutory regulation or exceeds the permitted use, you will need to obtain permission directly from the copyright holder. To view a copy of this licence, visit http://creativecommons. org/licenses/by/4.0/. 


\section{References}

Beeby S, White N (2010) Energy harvesting for autonomous systems. Artech House

Blomshield F (2007) Lessons learned in solid rocket combustion instability. In: 43rd AIAA/ASME/SAE/ASEE Joint Propulsion Conference \& Exhibit. https://doi.org/10.2514/6.2007-5803

Gere JM, Timoshenko SP (1997) Mechanics of materials. PWS Publishing Company

Kulkarni HR, Shukla SN, Dongare MB (2017) Determination of young's modulus of aluminium, copper, iron, brass and steel alloys by using double exposure holographic interferometry (Dehi) technique. Mater Sci Res India 14(2):128-132 https://doi. org $/ 10.13005 / \mathrm{msri} / 140207$

Kumar S, Srivastava R, Srivastava R (2016) Design and analysis of smart piezo cantilever beam for energy harvesting. Ferroelectrics 505(1):159-183. https://doi.org/10.1080/00150193.2016. 1255848

Makihara K, Onoda J, Minesugi K (2006) Novel attenuation method of transmitted sound into rocket faring using energy-harvesting technique. In: 47th AIAA/ASME/ASCE/AHS/ASC structures, structural dynamics, and materials conference 14th AIAA/ ASME/AHS Adaptive Structures Conference 7th. https://doi. org/10.2514/6.2006-2104

Makihara K, Shimose S (2012) Supersonic flutter utilization for effective energy-harvesting based on piezoelectric switching control. Smart Mater Res 2012:1-10. https://doi.org/10.1155/ $2012 / 181645$
Price EW (1959) Combustion instability in solid propellant rocket motors. In: IXth International Astronautical Congress/IX. Internationaler Astronautischer Kongress/IXe Con-grs International D'astronautique, pp 865-874 . https://doi.org/10.1007/978-37091-4745-0_26

Priya S, Inman DJ (2010) Energy harvesting technologies. Springer, New York

Safaei M, Sodano HA, Anton SR (2019) A review of energy harvesting using piezoelectric materials: state-of-the-art a decade later (2008-2018). Smart Mater Struct 28(11):113001. https:// doi.org/10.1088/1361-665x/ab36e4

Sutton GP, Biblarz O (2017) Rocket propulsion elements. Wiley, New York

Taherinezhad R, Zarepour G (2020) Evaluation of pressure oscillations by a laboratory motor. Chin J Aeronaut 33(3):805-825. https://doi.org/10.1016/j.cja.2019.11.010

Uddin MN, Islam, MS, Sampe J, Ali SHM, Bhuyan M (2016) Design and simulation of piezoelectric cantilever beam based on mechanical vibration for energy harvesting application. In: 2016 International Conference on innovations in science, engineering and technology (ICISET), pp 1-4. IEEE

$\mathrm{Xu}$ TB (2016) Energy harvesting using piezoelectric materials in aerospace structures. Structural Health Monitoring (SHM) in Aerospace Structures, pp 175-212. https://doi.org/10.1016/b9780-08-100148-6.00007-x

Publisher's Note Springer Nature remains neutral with regard to jurisdictional claims in published maps and institutional affiliations. 